\title{
Effect of Herbal Mycotoxin Binders in Amelioration of Induced Mycotoxicosis in White Leghorn Laying Hens
}

\author{
Swathi A, Jagadeesh S. Sanganal ${ }^{\star}$, Shridhar N.B, Rashmi R, Prem Kumar G and Suhasini K
}

Department of Veterinary Pharmacology and Toxicology, Veterinary College, KVAFSU, Hebbal, Bangalore, India

\begin{tabular}{|c|c|}
\hline $\begin{array}{ll}\text { Abstract } \\
\end{array}$ & Article Information \\
\hline \multirow{11}{*}{$\begin{array}{l}\text { Efficacy of herbal mycotoxin binders in ameliorating induced mycotoxicosis was evaluated in } \\
\text { white leghorn laying hens. Birds were randomly divided into six groups containing } 15 \text { birds in } \\
\text { each group. Group I was served as control fed with basal diet, group II birds were fed with } \\
\text { aflatoxins and ochratoxin A at } 100 \mathrm{ppb} \text { each. Group III, IV, V and VI birds were fed with } \\
\text { aflatoxins and ochratoxin A at } 100 \mathrm{ppb} \text { each and herbal mycotoxin binders Vilocym }{ }^{\circledR} \text {, } \\
\text { Toxiroak }^{\circledR} \text {, Vilocym- } \mathrm{Z}^{\circledR} \text { in feed at } 1 \mathrm{~kg} / \text { tonne and } \mathrm{AV} / \mathrm{LBP} / 20^{\circledR} \text { at } 1 \mathrm{ml} / \text { litre in drinking water } \\
\text { respectively for } 10 \text { weeks. The cultured rice and wheat samples were screened for presence } \\
\text { of mycotoxins by LC-MS/MS method. Aflatoxins concentration in cultured rice sample was } \\
826 \mathrm{ppb} \text {. Ochratoxin A concentration in cultured wheat sample was } 8990 \mathrm{ppb} \text {. The } \\
\text { hematological parametes viz., Hb, TEC, PCV showed signmificant decreased level in (Group } \\
\text { II, III, IV, V and VI) compared to their respective control group. Similarly biochemical } \\
\text { parameters viz., aspartate aminotransferase, alanine aminotransferase showed significantly } \\
\text { decreased level in treatred groups (Group II, III, IV, V and VI) compared to their respective } \\
\text { control group. Serum albumin and serum total protein level significantly decreased in treatred } \\
\text { groups (Group II, III, IV, V and VI) compared to their respective control group(Group I). } \\
\text { Histopathology of Group II birds revealed toxic effects on liver and kidney. Supplementation } \\
\text { of herbal mycotoxin binders in mycotoxicated feed showed improvement in all the parameters } \\
\text { indicating that herbal mycotoxin binders reduce the severity of toxicity. }\end{array}$} & listory: \\
\hline & Received : 06-06-2014 \\
\hline & Revised : $: 25-09-2014$ \\
\hline & Accepted : 2 \\
\hline & Is: \\
\hline & ig hens \\
\hline & Aspergillus flavus \\
\hline & Aspergillus ocl \\
\hline & kins \& oc \\
\hline & Herbal mycotc \\
\hline & $\begin{array}{l}{ }^{*} \text { Corresponding Author: } \\
\text { Jagadeesh S. Sanganal }\end{array}$ \\
\hline & \\
\hline
\end{tabular}

\section{INTRODUCTION}

Poultry industry which provides cheap source of animal protein has taken a quantum leap in the last three decades evolving from a near backyard practice to a venture of industrial promotion. Poultry is one of the fastest growing segments of the agricultural sector in India with rising rate of 8 per cent per annum and it is the major contributor of growing economy of the country. India stands fifth in the world egg producing countries with 55.6 billion eggs production during 2008. Therefore, the problems of feed related disorders have been given considerable attention in recent years. The quality of feed and feed ingredients plays an important role in determining the performance of birds and profit margin to the poultry farmers. In tropical countries like India, prevalence of high moisture and humid condition, poor harvesting techniques, inadequate drying facilities and unseasonal rains have provided conductive environment for fungal invasion of feed resulting in proliferation and elaboration of toxic metabolites called as mycotoxins. Among mycotoxins, aflatoxin and ochratoxin are the most common and simultaneous contaminants of feed stuffs. Aflatoxins (AF) are potent hepatotoxic and immunosuppressive agents produced by Aspergillus parasiticus and Aspergillus flavus. Aflatoxicosis is an acute or chronic condition characterized by growth retardation, reduced feed consumption, decreased body weight and immune suppression, decreased egg quality and production (Ghosh et al., 1990). Ochratoxins (OA) are a group of structurally related toxic fungal metabolites, which are potent nephrotoxic agents chiefly produced by Aspergillus ochraceus. Ochratoxicosis resulting from the ingestion of ochratoxin is an acute or chronic condition characterized by decrease in production performance, egg quality, growth retardation, decreased feed consumption, immune suppression and mortality (Sawale et al., 2009). AF and OA could interact synergistically and adversely affect the performance and productivity of birds.

Efforts have been made in the past to counteract the toxic effects of these toxins in the feed by use of physical, chemical and biological methods with limited success. Deleterious effects of aflatoxin could be overcome, or at least diminished, by adsorbents (Raju and Devegowda, 2000). Stoev et al.2000 and Kurkure et al. (2000) reported ameliorating effect of Artichoke and Curcuma longa (turmeric) powder on ochratoxin A and aflatoxin B1. However, published literature on protective role of herbal toxin binders in mycotoxicosis induced by combination of 
AF and $O A$ is scanty. Henceforth, present study was designed to study the protective role of four herbal mycotoxin binder products in reduction of mycotoxicosis induced by combined effect of AF and OA in white leghorn laying hens for ten week.

\section{MATERIALS AND METHODS}

Aspergillus flavus (MTCC 2798) and Aspergillus ochraceus (MTCC 10276) cultures were obtained from Microbial Type Culture Collection and Gene Bank (MTCC), Institute of Microbial Technology (IMTECH), Chandigarh, India. Potato Dextrose Agar (PDA) was procured from Himedia (Mumbai, India). Acetonitrile, formic acid, n- hexane, ammonium acetate buffer and methanol were used of HPLC grade Merck Chemicals. The mycotoxin standards like aflatoxins (B1, B2, G1 and G2), ochratoxin A, HT-2 toxin, diacetoxyscirpenol (DAS), deoxynivalenone (DON), 3-acetyldeoxynivalenol (3AcDON), 15-acetyldeoxynivalenol (15-AcDON), beauvericin, apicidin and citrinin were obtained from Sigma Aldrich Chemicals (Mumbai, India). T-2 toxin, fumonisin B1, fumonisin B2 and zearalenone were also used (Ermentek, Israel).

Aflatoxin was produced on polished rice using A.flavus (MTCC 2798) culture suggested by Shotwell et al. (1966). Ochratoxin was produced on wheat using A.ochraceus (MTCC 10276) culture suggested by Trenk et al. (1971). The rice was soaked in water for two hours and water for overnight and autoclaved at $15 \mathrm{psi}$ for $15 \mathrm{~min}$ and then each was inoculated with $0.5 \mathrm{ml}$ of respective fungal spore suspension. The inoculum was incubated for seven days at room temperature in dark place with vigorous shaking from third day after inoculation. On seventh day, the flasks with green coloured mycelial growth were autoclaved at $121^{\circ} \mathrm{C}$ (15 psi) for $20 \mathrm{~min}$. The fermented rice and wheat was collected and dried overnight in a hot air oven at $80^{\circ} \mathrm{C}$. The dried material was powdered and stored in a dark place.

\section{Extraction of Toxin}

Twenty one days old fungal cultured rice and wheat material was ground to fine powder in mixer and dried overnight in hot air oven at $60^{\circ} \mathrm{C}$. After that, $5 \mathrm{~g}$ of sample was extracted for six hour with $25.00 \mathrm{~mL}$ mixture of Acetonitrile/formic acid in water/hexane $(80: 19: 01)$ at $25^{\circ} \mathrm{C}$ under constant agitation. Extract was spun for 1:00 hour and the lower aqueous Acetonitrile layer $(4.00 \mathrm{~mL})$ was dried in a water bath under nitrogen streaming. The sample was dispersed in $4.00 \mathrm{~mL} 10 \mathrm{mM}$ ammonium acetate buffer $(\mathrm{pH} 8.5) /$ methanol (40:60) mixture. The sample $(2.00 \mathrm{~mL})$ was cleaned up using OASIS® - HLB cleanup SPE cartridge. The purified sample was dried and reconstituted in $0.500 \mathrm{~mL}$ of HPLC mobile phase $(10 \mathrm{mM}$ ammonium acetate $(\mathrm{pH} 8.5) /$ methanol $(40: 60 \% \mathrm{v} / \mathrm{v})$ and filtered through hydrophilic PTFE membrane syringe filters $(20 \mu \mathrm{m})$ to auto sampler vial.

\section{Analysis of Mycotoxins}

The extracts were analyzed for the presence of aflatoxins (B1, B2, G1 and G2), ochratoxin, trichothecenes, fumonisins, beauvericin, apicidin, zearalenone and citrinin by LC-MS/MS method. HPLC 1200 series (Agilent Technologies, Waldbron, Germany) coupled to Applied Biosystems (USA), MDS Sciex 3200 Q Trap LC-MS/MS System was used. HPLC column of Zorbax, Eclipse XDD-C 18, resolution, HT 4.6x50mm, 1.8micron, 600 Bar column was used (Agilent Technologies, Waldbron, Germany). The presence of 17 different mycotoxins was monitored using LC-MS/MS. Mycotoxin identity confirmation was performed by monitoring of at least two transitionions per analyte. The HPLC system utilized a binary gradient solvent system consisting of nanopure grade R0 water buffered by $10 \mathrm{mM}$ ammonium acetate $(\mathrm{pH} 8.5)$ and methanol (40:60\% $/ \mathrm{v})$. The total run time was 28 minutes per sample analyzed.

Quantification of toxins was carried out from standard calibration curve of each toxin. Calibration curves were built individually using 5 or 6 concentration levels in maize matrix (Mycotoxin free) for each mycotoxin analyzed. The peak area of the mycotoxins was plotted against the concentration to generate matrix matched calibration curve.

White leghorn laying hens (BV-300) of 25 weeks age were procured from a private poultry farm. Birds were acclimatized for one week and then randomly divided into six groups containing 15 birds in each group. Vaccination was done as per routine farm practice. Layer mash feed was procured from Department of Poultry Science, Veterinary College, KVAFSU, Hebbal, Bangalore, India. The feed sample was tested for mycotoxin levels before feeding to birds.

Powdered $\mathrm{AF}$ and $\mathrm{OA}$ culture materials were incorporated into the feed so as to give final concentration of $100 \mathrm{ppb}$ each. Four different toxin binder formulations were obtained from Ayurvet limited, Himachal Pradesh, India and were mixed in the feed at the rate of one $\mathrm{kg} / \mathrm{per}$ tonne of feed. Four different natural toxin binders were as follows;

1. Toxiroak ${ }^{\circledR}$ - Allium sativum, Azadirachtaindica, Solanumnigrum, Emblicaofficinalis, Curcuma longa and hydrated soduum calcium aluminosilicates (HSCAS).

2. Vilocym- $Z^{\circledR}$ - Solanumnigrum, Citrulluscolocynthis, PhyllanthusemblicaandCurcuma longa.

3. Vilocym ${ }^{\circledR}$ - Solanumxanthocarpum, Emblicaofficinalis, Ashwagandha and Azadirachtaindica.

4. AV/LBP $/ 20^{\circledR}$-Azadirachtaindica, Andrographis- paniculata, Citrulluscolocynthisand Emblicaofficinalis.

Birds were feed as per the protocol shown in Table 1. Control consist of birds feed without toxins and toxin binder.

Table 1: Birds feed treatment with and without toxins or toxin binder

\begin{tabular}{clcccc}
\hline Groups & Feed Treatments & $\begin{array}{c}\text { Aflatoxin } \\
(\mathbf{p p b})\end{array}$ & $\begin{array}{c}\text { Ochratoxin } \\
(\mathbf{p p b})\end{array}$ & $\begin{array}{c}\text { Toxin Binder } \\
(\mathbf{k g} / \text { tone feed })\end{array}$ & $\begin{array}{c}\text { Number } \\
\text { of Birds }\end{array}$ \\
\hline I & Control & - & - & - & 15 \\
II & Toxins without binder & 100 & 100 & - & 15 \\
III & Toxins + Vilocyn $^{\circledR}$ & 100 & 100 & 1 & 15 \\
IV & Toxins + Toxiroak $^{\circledR}$ & 100 & 100 & 1 & 15 \\
V & Toxins + Vilocyn-Z $^{\circledR}$ & 100 & 100 & 1 & 15 \\
VI & Toxins + AV/LBP/20 $^{\circledR}$ & 100 & 100 & 1 & 15 \\
\hline
\end{tabular}


Haematology parameters like total erythrocyte count (TEC), haemoglobin, packed cell volume (PCV) were estimated using blood samples collectec in EDTA vials on day 0,30 and $60^{\text {th }}$ day of experiment. Serum bilchemical parameters like alanine amino transferase (ALT), aspartate amino transferase (AST), total protein and albumin were estimated from the serum samples collected on $0,30^{\text {th }}$ and $60^{\text {th }}$ day of experiment using semi automatic biochemical analyser (ARTOS, Bangalore). Egg production (per day/ group) was recorded till the end of experiment. Weekly representative egg samples from each group were ansalysed for shape index, shell thickness. Eggs were weighed daily till the end of the experiment. At the end of the experiment all birds were scrificied and necropsy was conducted on each carcass to observe any gross pathological changes. The representative tissue samples were processed for histopathology and stained with haematoxylin and eosin (Luna, 1968).

\section{Statistical Analysis}

Mean values and standard error of means were calculated and expressed as mean SEM. The data was analyzed by two-way ANOVA with Bonferroni post-tests using GraphPad Prism Trial version 5 for Windows, GraphPad Software, San Diego California USA, www.graphpad.com.

\section{RESULTS}

Mycotoxins like aflatoxin B1, B2, G1, G2 and ochratoxin $A$ were identified and quantified in cultured rice and wheat samples (Figure 1). Aflatoxin concentration in cultured rice sample was $826 \mathrm{ppb}$ (Table 2). Ochratoxin A concentration in cultured wheat sample was $8990 \mathrm{ppb}$ (Table 2). The feed material showed the presence of minimm concentration of aflatoxin B1, B2, G2 and ochratoxin A (Table 2). The observed clinical signs were dullness, ruffled feather, depression and occasional diarrhoea in group II birds. Haemoglobin concentration in Group II, III, V, VI was significantly $(P<0.01)(P<0.001)$ lower than the control group value (Table 3$)$. Packed cell volume in Group II, III, VI, V, VI was significantly $(P<0.05)$ $(P<0.01) \quad(P<0.001)$ lower than the control group value (Table 3). Total erythrocyte count in Group II, III, V, VI was significantly $(P<0.05)(P<0.01)(P<0.001)$ lower than the control group value (Table 3 ). Alanine amino transferase in Group II, III, IV, V and VI was significantly $(P<0.001)$ higher than the control group value (Table 4$)$. Aspartate amino transferase in Group II, III, IV and V was significantly $(P<0.001)$ higher than the control group value (Table 4). Serum albumin in Group II, III, IV, V and VI was significantly $(P<0.001)$ lower than the control group value (Table 5). Serum total protein in Group II, III, IV, V and VI was significantly $(P<0.01) \quad(P<0.001)$ lower than the control group value (Table 5). Average egg weight in Group II was significantly $(P<0.01)$ lower than the control group value (Table 6). Average egg production in Group II was significantly $(P<0.001)$ lower than the control group value (Table 7). Egg shell thickness and shape index of weekly representative samples from all the groups did not show any significant difference during the experiment (Table 8, 9). At autopsy, none of the treated groups showed any gross pathological in any of the organ except in group II liver was pale and swollen and kidney was congested and swollen. Microscopically liver from toxin fed group revealed congestion and increase in sinusoidal space, separation of hepatic cords,focal infiltration of inflammatory cells in interstitium (Figure 2.2). kidney revealed congestion and haemorrhage, swollen tubular epithelial cells with granular eosinophilic cytoplasm, vacuolar degeneration, infiltration of inflammatory cells, mild interstitial connective tissue proliferation, desquamation of epithelial cells, complete degeneration and disappearance of glomeruli (Figure 2.4).

Table 2: Identification and quantification of different mycotoxin in fermented rice, fermented wheat and feed samples ( $\mu \mathrm{g} / \mathrm{kg}$ or $\mathrm{ppb}$ )

\begin{tabular}{lccc}
\hline Mycotoxins & \multicolumn{3}{c}{ Samples } \\
\cline { 2 - 4 } & $\begin{array}{c}\text { Fermented } \\
\text { Rice } \\
\text { Powder }\end{array}$ & $\begin{array}{c}\text { Fermented } \\
\text { Wheat } \\
\text { Powder }\end{array}$ & $\begin{array}{c}\text { Layer } \\
\text { Mash } \\
\text { Feed }\end{array}$ \\
\hline Aflatoxin B1 & 800.00 & 0.83 & 0.69 \\
Aflatoxin B2 & 21.40 & 1.95 & 1.64 \\
Aflatoxin G1 & 5.68 & 4.90 & NP \\
Aflatoxin G2 & 0.66 & 8.69 & 0.91 \\
Ochratoxin A & 35.50 & 8990 & 1.12 \\
T-2 Toxin & NP & NP & NP \\
HT-2 Toxin & NP & NP & NP \\
DAS & NP & NP & NP \\
DON & NP & NP & NP \\
3Ac-DON & NP & NP & NP \\
15-AcDON & NP & NP & NP \\
Fumonisin B1 & NP & NP & NP \\
Fumonisin B2 & NP & NP & NP \\
Zearalenone & NP & NP & NP \\
Citrinin & NP & NP & NP \\
Beauvericin & NP & NP & NP \\
Apicidin & NP & NP & NP \\
\hline & NP: No Peak &
\end{tabular}

Table 3: Effect of feed treatment with and without toxin and toxin binders on hematological parameters of white leghorn laying hens

\begin{tabular}{|c|c|c|c|c|c|c|c|c|c|}
\hline \multirow[t]{2}{*}{ Groups } & \multicolumn{3}{|c|}{ Hemoglobin (g\%) } & \multicolumn{3}{|c|}{ Packed Cell Volume (\%) } & \multicolumn{3}{|c|}{$\begin{array}{c}\text { Total Erythrocyte Count } \\
\text { (millions } / \mu \mathrm{L})\end{array}$} \\
\hline & Day 0 & Day 30 & Day 60 & Day 0 & Day 30 & Day 60 & Day 0 & Day 30 & Day 60 \\
\hline Group I & $8.3 \pm 0.1$ & $9.2 \pm 0.1$ & $11.1 \pm 0.3$ & $35.7 \pm 0.6$ & $30.7 \pm 1.3$ & $34.8 \pm 0.8$ & $2.5 \pm 0.1$ & $2.3 \pm 0.1$ & $2.1 \pm 0.0$ \\
\hline Group II & $8.3 \pm 0.2$ & $8.1 \pm 0.2^{* \star}$ & $8.0 \pm 0.2^{* * *}$ & $33.3 \pm 1.5$ & $29.7 \pm 1.3$ & $25.0 \pm 1.1^{\star * *}$ & $2.6 \pm 0.2$ & $1.9 \pm 0.1^{* \star}$ & $1.6 \pm 0.1^{\star \star \star}$ \\
\hline Group III & $8.4 \pm 0.1$ & $9.5 \pm 0.3$ & $10.2 \pm 0.2^{* *}$ & $36.0 \pm 0.9$ & $33.2 \pm 0.9$ & $30.3 \pm 1.6^{*}$ & $2.4 \pm 0.1$ & $2.1 \pm 0.0$ & $1.8 \pm 0.1^{*}$ \\
\hline Group IV & $8.3 \pm 0.1$ & $9.0 \pm 0.1$ & $10.7 \pm 0.1$ & $35.8 \pm 0.9$ & $33.0 \pm 0.9$ & $29.3 \pm 0.7^{\star \star}$ & $2.4 \pm 0.1$ & $2.2 \pm 0.1$ & $1.9 \pm 0.1$ \\
\hline Group V & $8.5 \pm 0.2$ & $8.4 \pm 0.1^{\star *}$ & $8.3 \pm 0.1^{* * *}$ & $35.7 \pm 1.2$ & $28.7 \pm 1.7$ & $25.0 \pm 1.3^{\star * *}$ & $2.5 \pm 0.1$ & $2.3 \pm 0.1$ & $1.8 \pm 0.1^{*}$ \\
\hline Group VI & $8.3 \pm 0.1$ & $11.0 \pm 0.2^{* * *}$ & $9.1 \pm 0.1^{\star \star \star}$ & $34.2 \pm 1.2$ & $27.2 \pm 0.5$ & $22.3 \pm 0.5^{\star \star \star}$ & $2.4 \pm 0.1$ & $2.1 \pm 0.0$ & $1.8 \pm 0.1^{*}$ \\
\hline
\end{tabular}




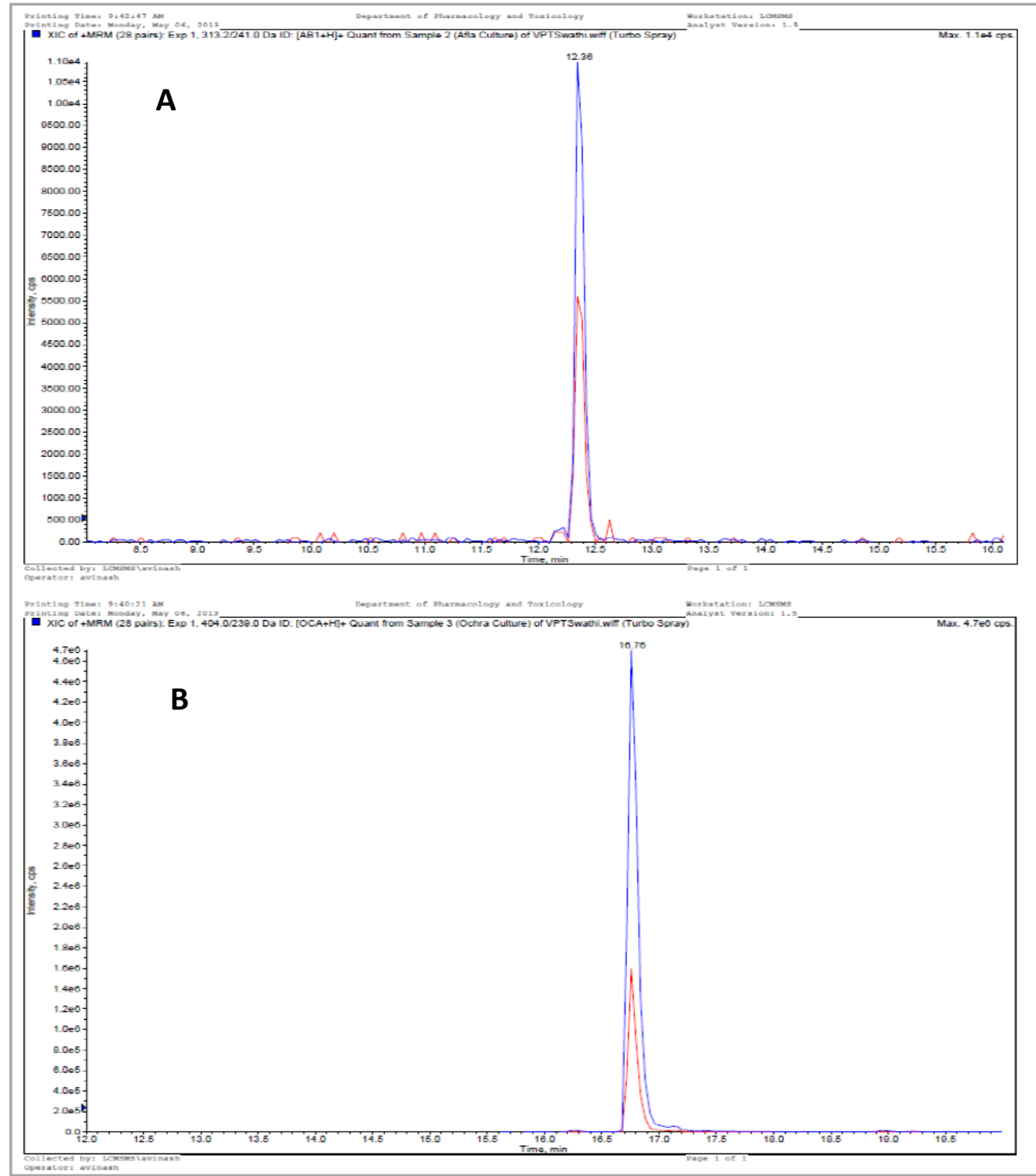

Figure 1: Extracted ion chromatogram for AFB1 (A) and ochratoxin A in rice and wheat sample respectively

Table 4: Effect of feed treatment with and without toxin and toxin binders on Alanine Amino Transferase (ALT) and Aspartate Amino Transferase (AST) level of white leghorn laying hens

\begin{tabular}{|c|c|c|c|c|c|c|}
\hline \multirow[b]{2}{*}{ Groups } & \multicolumn{3}{|c|}{ ALT ( U/L) } & \multicolumn{3}{|c|}{ AST ( U/L) } \\
\hline & Day 0 & Day 30 & Day 60 & Day 0 & Day 30 & Day 60 \\
\hline Group I & $24.2 \pm 0.9$ & $24.1 \pm 0.9$ & $23.2 \pm 0.5$ & $93.2 \pm 2.2$ & $119.6 \pm 1.6$ & $124.1 \pm 1.2$ \\
\hline Group II & $25.5 \pm 1.0$ & $38.7 \pm 0.8^{\star \star *}$ & $52.6 \pm 2.4^{\star * *}$ & $95.3 \pm 1.5$ & $167.4 \pm 10.0^{\star * *}$ & $191.9 \pm 6.3^{* * *}$ \\
\hline Group III & $24.3 \pm 0.5$ & $33.0 \pm 0.9^{* * *}$ & $43.2 \pm 2.0^{\star \star *}$ & $97.7 \pm 1.0$ & $134.7 \pm 2.2$ & $153.9 \pm 10.5^{\star * *}$ \\
\hline Group IV & $25.1 \pm 0.7$ & $34.0 \pm 1.3^{\star * \star}$ & $40.6 \pm 1.5^{\star \star \star}$ & $96.5 \pm 1.2$ & $125.0 \pm 1.4$ & $151.5 \pm 8.0^{* * *}$ \\
\hline Group V & $23.9 \pm 0.4$ & $34.2 \pm 1.6^{* * *}$ & $41.1 \pm 2.5^{\star \star *}$ & $98.4 \pm 0.4$ & $122.1 \pm 2.2$ & $167.5 \pm 10.2^{* * \star}$ \\
\hline Group VI & $25.3 \pm 0.5$ & $35.8 \pm 1.1^{* * *}$ & $40.8 \pm 1.6^{* * *}$ & $97.2 \pm 1.0$ & $113.4 \pm 4.0$ & $138.7 \pm 4.1$ \\
\hline
\end{tabular}

Table 5: Effect of feed treatment with and without toxin and toxin binders on serum albumin and total protein level of white leghorn laying hens

\begin{tabular}{|c|c|c|c|c|c|c|}
\hline \multirow{2}{*}{ Groups } & \multicolumn{3}{|c|}{ Serum albumin (g/dL) } & \multicolumn{3}{|c|}{ Serum total protein $(\mathrm{g} / \mathrm{dL})$} \\
\hline & Day 0 & Day 30 & Day 60 & Day 0 & Day 30 & Day 60 \\
\hline Group-I & $3.3 \pm 0.1$ & $3.3 \pm 0.1$ & $3.3 \pm 0.0$ & $5.3 \pm 0.1$ & $5.1 \pm 0.1$ & $5.0 \pm 0.2$ \\
\hline Group-II & $3.1 \pm 0.1$ & $2.4 \pm 0.1^{* * *}$ & $2.7 \pm 0.1^{\star \star \star}$ & $5.5 \pm 0.1$ & $4.3 \pm 0.1^{* \star *}$ & $3.7 \pm 0.2^{* \star *}$ \\
\hline Group-III & $3.3 \pm 0.1$ & $3.0 \pm 0.0$ & $2.8 \pm 0.0^{\star \star \star}$ & $5.2 \pm 0.0$ & $4.6 \pm 0.1^{\star \star}$ & $3.8 \pm 0.2^{\star \star \star}$ \\
\hline Group-IV & $3.4 \pm 0.2$ & $3.0 \pm 0.1$ & $2.5 \pm 0.1^{\star \star *}$ & $5.2 \pm 0.0$ & $4.5 \pm 0.1^{* * *}$ & $4.4 \pm 0.2^{* * *}$ \\
\hline Group-V & $3.2 \pm 0.1$ & $3.5 \pm 0.1$ & $2.9 \pm 0.1 * *$ & $5.6 \pm 0.1$ & $4.6 \pm 0.1^{\star *}$ & $4.0 \pm 0.1^{* * *}$ \\
\hline Group-VI & $3.3 \pm 0.1$ & $3.0 \pm 0.1$ & $2.2 \pm 0.2^{\star \star \star}$ & $5.5 \pm 0.1$ & $4.6 \pm 0.1^{* *}$ & $3.7 \pm 0.1^{* * *}$ \\
\hline
\end{tabular}


Table 6: Effect of feed treatment with and without toxin and toxin binders on average egg weight $(\mathrm{g})$ of white leghorn laying hens

\begin{tabular}{|c|c|c|c|c|c|c|}
\hline \multirow[b]{2}{*}{ Week } & \multicolumn{6}{|c|}{ Group } \\
\hline & $I$ & II & III & IV & $\mathbf{V}$ & VI \\
\hline 1 & $54.23 \pm 1.31$ & $52.66 \pm 3.11$ & $56.62 \pm 2.52$ & $52.16 \pm 1.37$ & $52.66 \pm 2.95$ & $54.16 \pm 1.04$ \\
\hline 2 & $51.00 \pm 0.89$ & $55.00 \pm 2.67$ & $51.66 \pm 2.07$ & $50.66 \pm 2.91$ & $54.66 \pm 1.60$ & $51.33 \pm 2.17$ \\
\hline 3 & $48.16 \pm 1.93$ & $52.16 \pm 2.33$ & $52.66 \pm 2.55$ & $53.83 \pm 1.99$ & $52.66 \pm 1.49$ & $46.66 \pm 2.06$ \\
\hline 4 & $53.16 \pm 2.34$ & $50.16 \pm 1.26$ & $55.05 \pm 0.90$ & $48.13 \pm 3.45$ & $51.41 \pm 1.54$ & $52.33 \pm 1.89$ \\
\hline 5 & $48.66 \pm 0.84$ & $49.86 \pm 1.60$ & $54.58 \pm 1.68$ & $51.81 \pm 2.03$ & $53.08 \pm 1.07$ & $52.80 \pm 1.33$ \\
\hline 6 & $53.00 \pm 1.00$ & $53.90 \pm 1.78$ & $55.10 \pm 1.66$ & $53.68 \pm 1.63$ & $54.50 \pm 0.61$ & $52.85 \pm 1.85$ \\
\hline 7 & $52.66 \pm 1.25$ & $49.71 \pm 1.91$ & $54.30 \pm 1.95$ & $54.73 \pm 0.83$ & $54.81 \pm 0.71$ & $51.85 \pm 1.10$ \\
\hline 8 & $51.00 \pm 1.52$ & $56.25 \pm 1.61$ & $53.20 \pm 1.44$ & $53.08 \pm 1.62$ & $56.38 \pm 0.40$ & $55.93 \pm 1.30$ \\
\hline 9 & $55.50 \pm 1.20$ & $56.10 \pm 2.62$ & $54.88 \pm 1.35$ & $54.91 \pm 1.09$ & $57.75 \pm 0.92$ & $54.73 \pm 1.08$ \\
\hline 10 & $58.00 \pm 1.36$ & $50.71 \pm 2.91^{*}$ & $54.33 \pm 2.51$ & $56.70 \pm 0.85$ & $57.95 \pm 0.99$ & $56.83 \pm 1.51$ \\
\hline
\end{tabular}

Table 7: Effect of feed treatment with and without toxin and toxin binders on average egg production (per day/group) of white leghorn laying hens

\begin{tabular}{|c|c|c|c|c|c|c|}
\hline \multirow[b]{2}{*}{ Week } & \multicolumn{6}{|c|}{ Group } \\
\hline & I & II & III & IV & $\mathbf{V}$ & VI \\
\hline 1 & $6.93 \pm 0.06$ & $6.93 \pm 0.06$ & $7.00 \pm 0.00$ & $6.80 \pm 0.10$ & $7.00 \pm 0.00$ & $7.00 \pm 0.00$ \\
\hline 2 & $6.93 \pm 0.06$ & $6.80 \pm 0.10$ & $6.53 \pm 0.19$ & $6.40 \pm 0.21$ & $6.73 \pm 0.15$ & $6.40 \pm 0.23$ \\
\hline 3 & $6.80 \pm 0.10$ & $6.40 \pm 0.21$ & $6.60 \pm 0.16$ & $6.73 \pm 0.11$ & $6.45 \pm 0.23$ & $6.60 \pm 0.19$ \\
\hline 4 & $6.80 \pm 0.10$ & $6.46 \pm 0.19$ & $6.60 \pm 0.19$ & $6.53 \pm 0.53$ & $6.23 \pm 0.23$ & $6.66 \pm 0.12$ \\
\hline 5 & $6.53 \pm 0.13$ & $6.73 \pm 0.11$ & $6.20 \pm 0.14$ & $6.26 \pm 0.11$ & $6.53 \pm 0.16$ & $6.33 \pm 0.18$ \\
\hline 6 & $6.60 \pm 0.13$ & $6.46 \pm 0.16$ & $6.60 \pm 0.13$ & $6.80 \pm 0.10$ & $6.86 \pm 0.14$ & $6.93 \pm 0.60$ \\
\hline 7 & $6.46 \pm 0.13$ & $6.06 \pm 0.11$ & $6.66 \pm 0.12$ & $6.26 \pm 0.22$ & $6.83 \pm 0.16$ & $6.46 \pm 0.13$ \\
\hline 8 & $6.13 \pm 0.21$ & $5.73 \pm 0.18$ & $6.73 \pm 0.18$ & $6.40 \pm 0.21$ & $6.63 \pm 0.13$ & $6.13 \pm 0.19$ \\
\hline 9 & $6.06 \pm 0.26$ & $5.13 \pm 0.09^{* * *}$ & $6.00 \pm 0.13$ & $6.06 \pm 0.20$ & $6.53 \pm 0.13$ & $5.46 \pm 0.13$ \\
\hline 10 & $6.00 \pm 0.30$ & $4.66 \pm 0.23^{\star * *}$ & $6.33 \pm 0.18$ & $5.73 \pm 0.20$ & $6.26 \pm 0.11$ & $5.23 \pm 0.11^{*}$ \\
\hline
\end{tabular}

Table 8: Effect of feed treatment with and without toxin and toxin binders on shell thickness (mm) of weekly representative samples of white leghorn laying hens

\begin{tabular}{|c|c|c|c|c|c|c|}
\hline \multirow{2}{*}{ Week } & \multicolumn{6}{|c|}{ Group } \\
\hline & I & II & III & IV & $\mathbf{V}$ & VI \\
\hline 1 & $0.24 \pm 0.02$ & $0.18 \pm 0.01$ & $0.22 \pm 0.02$ & $0.25 \pm 0.04$ & $0.21 \pm 0.01$ & $0.21 \pm 0.01$ \\
\hline 2 & $0.24 \pm 0.04$ & $0.21 \pm 0.02$ & $0.26 \pm 0.01$ & $0.20 \pm 0.02$ & $0.26 \pm 0.02$ & $0.27 \pm 0.04$ \\
\hline 3 & $0.24 \pm 0.02$ & $0.20 \pm 0.02$ & $0.23 \pm 0.04$ & $0.18 \pm 0.01$ & $0.19 \pm 0.01$ & $0.24 \pm 0.02$ \\
\hline 4 & $0.26 \pm 0.03$ & $0.18 \pm 0.01$ & $0.27 \pm 0.01$ & $0.19 \pm 0.0$ & $0.23 \pm 0.04$ & $0.26 \pm 0.03$ \\
\hline 5 & $0.19 \pm 0.02$ & $0.21 \pm 0.02$ & $0.25 \pm 0.02$ & $0.25 \pm 0.02$ & $0.25 \pm 0.02$ & $0.21 \pm 0.01$ \\
\hline 6 & $0.25 \pm 0.02$ & $0.21 \pm 0.03$ & $0.25 \pm 0.03$ & $0.26 \pm 0.01$ & $0.23 \pm 0.02$ & $0.27 \pm 0.02$ \\
\hline 7 & $0.21 \pm 0.01$ & $0.25 \pm 0.04$ & $0.22 \pm 0.04$ & $0.27 \pm 0.04$ & $0.18 \pm 0.01$ & $0.19 \pm 0.02$ \\
\hline 8 & $0.18 \pm 0.01$ & $0.25 \pm 0.01$ & $0.26 \pm 0.01$ & $0.23 \pm 0.01$ & $0.18 \pm 0.01$ & $0.20 \pm 0.01$ \\
\hline 9 & $0.21 \pm 0.02$ & $0.22 \pm 0.03$ & $0.24 \pm 0.04$ & $0.22 \pm 0.04$ & $0.25 \pm 0.03$ & $0.25 \pm 0.02$ \\
\hline 10 & $0.25 \pm 0.02$ & $0.19 \pm 0.02$ & $0.26 \pm 0.01$ & $0.24 \pm 0.01$ & $0.18 \pm 0.01$ & $0.20 \pm 0.02$ \\
\hline
\end{tabular}

Table 9: Effect of feed treatment with and without toxin and toxin binders on shape index of weekly representative samples of white leghorn laying hens

\begin{tabular}{ccccccc}
\hline \multirow{2}{*}{ Week } & \multicolumn{6}{c}{ Group } \\
\cline { 2 - 7 } & I & II & III & IV & V & VI \\
\hline 1 & $77.61 \pm 0.81$ & $77.32 \pm 0.31$ & $78.53 \pm 0.62$ & $77.57 \pm 1.19$ & $78.74 \pm 0.56$ & $78.17 \pm 0.39$ \\
2 & $78.38 \pm 1.11$ & $76.76 \pm 0.47$ & $77.22 \pm 0.23$ & $78.53 \pm 0.97$ & $77.50 \pm 0.50$ & $78.28 \pm 0.87$ \\
3 & $77.52 \pm 1.06$ & $78.04 \pm 1.03$ & $77.90 \pm 0.53$ & $78.57 \pm 0.61$ & $76.13 \pm 0.63$ & $77.22 \pm 0.74$ \\
4 & $77.32 \pm 0.66$ & $77.57 \pm 1.19$ & $78.74 \pm 0.56$ & $78.09 \pm 0.87$ & $77.50 \pm 0.50$ & $78.28 \pm 0.87$ \\
5 & $77.67 \pm 0.62$ & $78.53 \pm 0.97$ & $77.50 \pm 0.50$ & $76.85 \pm 0.67$ & $77.50 \pm 0.50$ & $78.28 \pm 0.87$ \\
6 & $76.42 \pm 0.77$ & $78.57 \pm 0.61$ & $76.13 \pm 0.63$ & $77.22 \pm 0.48$ & $77.50 \pm 0.75$ & $78.82 \pm 0.93$ \\
7 & $78.53 \pm 0.62$ & $77.57 \pm 1.19$ & $78.74 \pm 0.56$ & $77.76 \pm 0.70$ & $77.33 \pm 0.68$ & $77.64 \pm 0.82$ \\
8 & $77.22 \pm 0.23$ & $78.53 \pm 0.97$ & $77.50 \pm 0.50$ & $76.50 \pm 0.41$ & $78.53 \pm 0.97$ & $78.61 \pm 0.87$ \\
9 & $77.90 \pm 0.53$ & $78.57 \pm 0.61$ & $76.13 \pm 0.63$ & $76.74 \pm 0.38$ & $76.74 \pm 0.38$ & $77.17 \pm 0.75$ \\
10 & $77.29 \pm 0.80$ & $79.27 \pm 0.54$ & $77.24 \pm 0.48$ & $77.64 \pm 0.60$ & $76.91 \pm 0.57$ & $78.85 \pm 0.85$ \\
\hline \multicolumn{7}{c}{ Values are Mean $\pm S E, \mathrm{n}=6{ }^{* \star *} P<0.001,{ }^{\star \star} P<0.01,{ }^{*} P<0.05$} \\
\end{tabular}



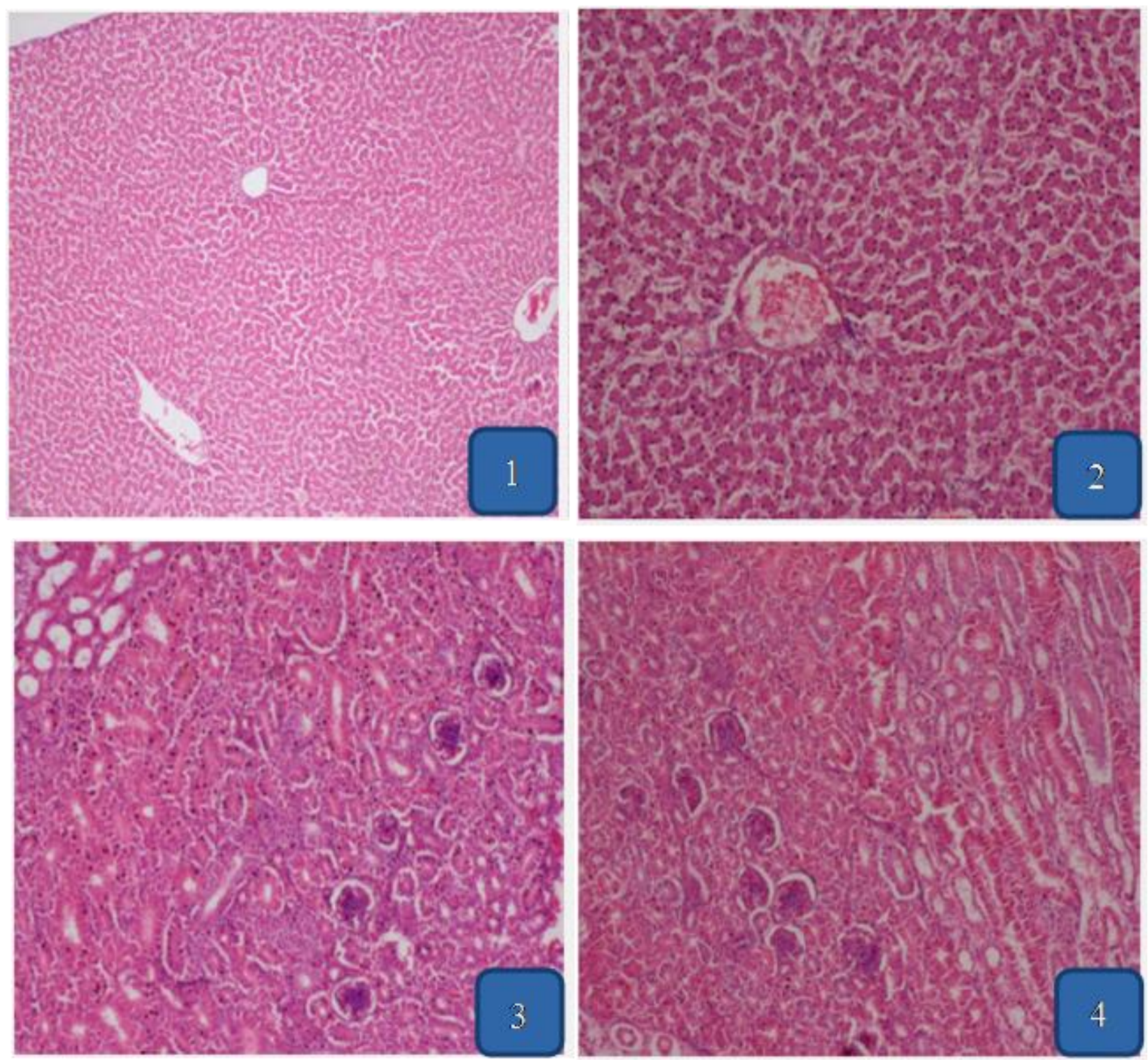

Figure 2: Microscopical sections of liver and kidney of Group I and II white leghorn laying hen, (1) liver section of Group I (2) liver section of Group II (3) kidney section of Group I and (4) kidney section of group II (H and E $10 \mathrm{X})$

\section{DISCUSSION}

The mycotoxicated birds showed ruffled feathers, dullness, depression and occasional diarrhoea. Observations are in concomitance with those reported by Sawale et al. (2009). The haemotalogical parametes showed significantly decreased level in treatred groups (Group II, III, IV, V and VI) compared to their respective control group (Group I). This was in accordance with the findings of Mani et al. (1993) and Ramadevi et al. (2000) who reported decrese in $\mathrm{Hb}$, TEC and PCV values in broilers fed with aflatoxin and ochrotoxin respectively. Improvement in haematological values were recorded in treatment groups supplemented with Vilocym $\AA$ in group III, Toxiroak ${ }^{\circledR}$ in group IV, Vilocym- ${ }^{\circledR}$ in $V$ and $\mathrm{AV} / \mathrm{LBP} / 20^{\circledR}$ in $\mathrm{VI}$ as compared to the mycotoxin fed group II during the experiment and the values are comparable with control group I fed with basal diet. These results are in correlation with earlier findings of Sawarkar et al. (2011) on $\mathrm{AF}$ and $\mathrm{OA}$ at $100 \mathrm{ppb}$ each in feed and its amelioration with Toxiroak Gold $^{\circledR}$ in broilers. Sawarkar et al. (2012) reported that broilers feed with $A F$ and $O A$ at $100 \mathrm{ppb}$ each in feed with Vilocym- $Z^{\circledR}$ in broilers showed lowered reduction in haematological values. The result indicated that herbal toxin binders could improve the haemotological parameters of leghorn hens by binding with toxin in the feed and making toxin less effective.

Serum albumin and serum total protein level significantly decreased in treatred groups (Group II, III, IV, $\mathrm{V}$ and $\mathrm{VI}$ ) compared to their respective control group (Group I). This was in accordance with the findings of Umar et al. (2012) who reported significant decrease in serum total protein values in broilers fed with aflatoxin and ochratoxin in feed at $3 \mathrm{mg} / \mathrm{kg}$ each for 6 weeks. Sawale et al. (2009) reported significant decrease in total serum protein and albumin in layers fed with ochratoxin $A$ at 1 ppm from 26 weeks age and its amelioration with Toxiroak $^{\circledR}$.

The biochemical parametes showed significantly decreased level in treatred groups (Group II, III, IV, V and VI) compared to their respective control group(Group I). This was in accordance with the findings of (Sawarkar et al. 2011 and 2012) who reported decrease in serum 
Swathi et al.,

protein concentration and increased ALT and AST level was reported in broilers fed with aflatoxins and ochratoxins at 100ppb each for 42 days and its amelioration with Toxiroak Gold® ${ }^{\circledR}$ and Vilocym- $Z^{\circledR}$. The result suggested that birds feed with herbal toxin binder could lowered biochemical changes in the leghorn laying hens owing to the effect of antioxidative activity of natural phytochemicals in herbal binders.

Egg shell thickness and shape index of weekly representative samples from all the groups did not show any significant difference during the experiment. Egg weight and egg production significantly decreased in Group II compared to control group. This was in accordance with Manafi and Khosravinia, (2013) who reported decreased egg production and egg weight in broiler breeders fed with $500 \mu \mathrm{g}$ aflatoxin B1/kg feed. This was also in concomitance with the findings of Sawale et al. (2009) reported similar results in average egg production in layers fed with ochratoxin at $1 \mathrm{ppm}$ and found improvement with Toxiroak ${ }^{\circledR}$ feed. Histopathology Gorup II birds revealed mycotoxin effects on liver and kidney. Microscopic lesion on liver are in agreement with report of Sawarkar et al. (2011) in broilers fed with aflatoxins and ochratoxins at $100 \mathrm{ppb}$ each and amelioration with Toxiroak Gold ${ }^{\circledR}$. Microscopic lesions on kidney are in agreement with report of Anitha 2007 in broilers fed with ochratoxins at $1 \mathrm{ppm}$ and amelioration with herbal oil and Toxiroak Gold ${ }^{\circledR}$. Degenerative change in tubules and glomeruli was also in accordance with the findlings of Mohiuddin et al. (1992) and Ramadevi et al. (1998).

\section{CONCLUSIONS}

Feeding aflatoxin and ochratoxin in combination significantly reduced egg weight, egg production, haematological parameters, total serum proteins, albumin and increased serum ALT and AST levels. In addition mycotoxin fed birds also showed microscopic lesions in liver and kidney, clinical signs of dullness, depression, diarrhoea and ruffled feathers indicating adverse effects on general health of layer chickens. Supplementation of herbal mycotoxin binders in mycotoxicated feed showed improvement in all the above discussed parameters indicating that herbal mycotoxin binders reduce the severity of toxicity. This would be helpful to the farming community to reduce ill effects of mycotoxins. Moreover, herbal toxin binders could be safe and natural source to avoid mycotoxicosis in animal.

\section{ACKNOWLEDGEMENTS}

Authors are thankful to Ayurvet Limited, Baddi, Himachal Pradesh, India for providing financial support and samples to conduct research.

\section{REFERENCES}

Anitha, N. (2007). Studies on ochratoxicosis and ameliorative affects of a toxin binder and herbal oil in broiler chicken.
Sci. Technol. Arts Res. J., July-Sep 2014, 3(3): 63-69

M.V.Sc. thesis.submitted to Karnataka Veterinary, Animal and Fisheries Sciences University, Bangalore.

Ghosh, J.D., Singh, J., Manipal, S.K. and Kulshreshtha, R.C. (1990). Effect of aflatoxin contaminated feeds on egg production and broiler growth. Indian Journal of Animal Sciences 60: 725-727.

Kurkure, N.V., Pawar, S.P., Kognole, S.M., Bhandarkar, A.G., Ganorkar, A.G. and Kalorey, D.R. (2000). Ameliorative effect of turmeric(Curcuma longa) in induced aflatoxicosis in cockerels. Indian Journal of Veterinary Pathology 24: 26-28.

Manafi, M. and Khosravinia, H. (2013). Effects of aflatoxin on the performance of broiler breeders and its alleviation through herbal mycotoxin binder. Journal of Agricultural Science and Technology 15: 55-63.

Mani, K., Narhari, D., Kumaraj, R. and Ramamoorthy, N. (1993). Influence of dietary aflatoxin B1 on certain haematological and biochemical characters of broiler chicken. Indian Veterinary Journal 70: 801-804.

Mohuddin, S.M., Vikram R. M. and Ahmed, S.R. (1992). Studies on ochratoxicosis in broiler chicks. Indian Veterinary Journal 69: 1011-1014.

Raju, M.V.L.N. and Devegowda, G. (2000). Influence of esterified glucomannan on performance and organ morphology, Serum biochemistry and haematology and broiler exposed to individual and combined mycotoxicosis (aflatoxin, ochratoxinand T-2 toxin). British Poultry Science 41: 640-650.

Ramadevi, N.R., Gopal N.R. and Ravikumar, P. (2000). An assessment of the protective effect of bentonite on ochratoxicosis in broiler with reference to certain haematological profile. Indian Veterinary Journal 77: 303306

Ramadevi, V., Gopal, N.R. and Sree Raman, P.K. (1998). Pathology of ochratoxicosis in broilers. Indian Journal of Veterinary Pathology 22: 93-95

Sawale, G.K., Gosh, R.C., Ravikanth, K., Maini, S. and Rekhe, D.S. (2009). Experimental mycotoxicosis in layer induced by ochratoxin $A$ and its amelioration with herbomineral toxin binder Toxiroak ${ }^{\circledR}$. International Journal of Poultry Science 8: 798-803.

Sawarkar, A.R., Saxena, M.J., Maini, S. and Ravikanth, K. (2012). Efficacy of herbomineral toxin binder 'Vilocym Z®' in amelioration of mixed mycotoxicosis in broilers. International Journal of Poultry Science 11: 209-216

Sawarkar, A.R., Sonkusale, P.M., Kurkure, N.V., Jangade, C.R., Maini, S. and Ravikanth, K. (2011). Experimental afla and ochratoxin induced mixed mycotoxicosis in broilers and its amelioration with herbomineral toxin binder 'Toxiroak Gold®'. International Journal of Poultry Science 10: 560-566.

Stoev, S.D., Anguelov, G., Ivanou, I. and Pavlov, D. (2000). Influence of ochratoxin A and an extract of artichoke on the vaccinal immunity and health in broiler chicks. Experimental and Toxicologic Pathology 52: 43-55

Umar, S., Arshad, A., Ahmad, B. and Arshad, M. (2012). Clinico biochemical and haematological changes in broilers induced by concurrent exposure to aflatoxin B1 and ochratoxin A. Journal of Public Health and Biological Sciences 1: 3. 